\title{
Advantages of Implantable Contact Lenses Over Laser-assisted In Situ Keratomileusis
}

a report by

Alaa EI-Danasoury

Medical Director, Magrabi Eye and Ear Hospital, Jeddah

Implantation of phakic intraocular lenses (IOLs) has many advantages over keratorefractive procedures such as laser-assisted in situ keratomileusis (LASIK). In the treatment of high-degree myopia by LASIK, relatively large amounts of cornea must be ablated, small effective optical zones are created and the predictability and stability of the procedure begin to diminish. ${ }^{1,2}$ Furthermore, many refractive surgeons have reconsidered the assumption that an alteration of the natural corneal shape is the best way to address myopic and astigmatic refractive errors based on a number of factors, including a better understanding of the biomechanical effect of LASIK on the cornea (especially in severe cases of myopia), the increasing number of reported corneal ectasia cases after LASIK and the development of sophisticated wavefront analysers that objectively measure the negative effect of LASIK on quality of vision (especially in relation to surgically induced high-order aberrations). ${ }^{3}$

The potential removability and exchangeability of phakic IOL implantation is generally absent in keratorefractive procedures. Co-existing astigmatism can be neutralised at the time of the surgery by varying the incision through which the lens is implanted or by the use of toric phakic IOLs. One of the most important advantages over techniques such as clear lens extraction with $\mathrm{IOL}$ implantation is the preservation of accommodation. Furthermore, lensectomy may increase the risk of retinal detachment and cystoid macular oedema, especially if neodyniumdoped: yttrium-aluminium-garnet (Nd:YAG) laser posterior capsulotomy is required for posterior capsule opacification. ${ }^{4,5}$

\section{Implantable Contact Lens}

Implantable contact lenses (ICLs) are phakic posterior chamber lenses manufactured by Staar Surgical (Nidau, Switzerland). They use a single-piece plate-haptic lens designed to anteriorly vault to the crystalline lens and intended to have minimal contact with the natural lens. The lens material is a proprietary hydrophilic collagen polymer - a co-polymer of $63 \%$ hydroxyethyl-methyl-acrylate, $0.2 \%$ collagen and $3.4 \%$ a benzofenone for ultraviolet (UV) absorption - known as Collamer ${ }^{\circledR}$, with a water content of $34 \%$, light transmission properties of $99 \%$ and a refractive index of 1.45 at $35^{\circ} \mathrm{C}$. Three versions of ICL are currently available. ${ }^{6}$

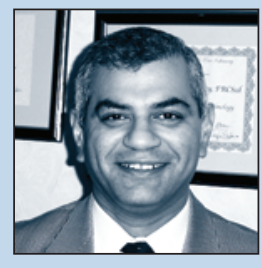

Alaa El-Danasoury is Medical Director of the Magrabi Ey and Ear Hospital in Jeddah and Director of the Refractive Surgery Institute at the same institution. He has written numerous scientific papers and edited textbooks related to refractive surgery, and has been instumental in the development and perfection of laser-assisted in situ keratomileusis technologies, phakic intraocular lenses and lamellar keratoplasty. He received fellowships in cornea and external eye diseases from the Royal College of Surgeons (RCS), Edinburgh and Emory College, Atlanta.

E: malaa@magrabi.com.sa
The myopic version of the ICL (V4 version) is plano-concave, with the anterior surface being plano; the optic diameter ranges from 4.65 to $5.50 \mathrm{~mm}$. The thickness of the lens is less than $50 \mu \mathrm{m}$ at the centre of the optic and less than $100 \mu \mathrm{m}$ at the haptic plate. The thickest part of the myopic $I C L$ is the junction between the optic and the haptic and ranges from 300 to $700 \mu \mathrm{m}$. The overall length of myopic ICL ranges from 11.5 to $13 \mathrm{~mm}$ in $0.5 \mathrm{~mm}$ increments. Power ranges from -23.00 to -3.00 diopters (D) in half-D increments. ${ }^{6-10}$ The hyperopic ICL (V3 version) is concave-convex, with the anterior surface being convex. The optic diameter is fixed at $5.5 \mathrm{~mm}$ for all powers, and the thickest part is the centre of the optic. Overall lengths of hyperopic ICLs range from 11 to $12.5 \mathrm{~mm}$ in $0.5 \mathrm{~mm}$ increments. Power ranges from +3.00 to $+21.50 \mathrm{D}$ in half- $\mathrm{D}$ increments. 6,10 The toric ICL (V4 version) has a toric convex-concave optic that incorporates the desired cylindrical power in a specific axis as required to correct a given patient's astigmatic condition. It is manufactured using the platform of the non-toric design and is similar to the spherical ICL in terms of size, thickness and configuration, with the addition of a toric optic to correct myopia with astigmatism. To minimise the rotation required by the surgeon during implantation, the toric ICL is custom-made to be implanted on the horizontal axis. The time from order to delivery for a toric ICL is between four and six weeks; to shorten this time, the surgeon has the option of using a ready-made toric $I C L$ of the same required power with a cylinder axis within $22.5^{\circ}$ of the power needed. In such cases, the 'alternative' toric ICL will have to be rotated inside the eye to compensate for the difference in axis orientation. Each toric ICL is sent to the surgeon with a guide that demonstrates the amount and direction of rotation from the horizontal axis required to align the toric $\mathrm{ICL}$ cylinder axis to correct the patient's astigmatism. It is recommended that rotation is less than $22.5^{\circ}$ from the horizontal. The cylindrical power ranges from 1.0 to $6.0 \mathrm{D}$ with the same range of spherical power as the myopic ICL.6,11,12

The myopic ICL (V4 version) was approved by the US Food and Drug Administration (FDA) in 1995 for the full range of powers between -20.00 and -3.00D. FDA studies for the toric and hyperopic ICL are under way. Any combination of the myopic and hyperopic versions with cylinder corrections can be customised. Clinical results published in the literature, as well as reports from FDA studies, demonstrate impressive results, including high predictability, efficacy, safety and stability and high levels of patient satisfaction after ICL and toric ICL implantation. Long-term followup on a large series has established the safety of the spherical ICL. ${ }^{7-12}$

\section{Implantable Contact Lenses versus Laser-assisted} In Situ Keratomileusis - Clinical Studies

\section{Myopic Correction}

Various clinical studies were designed to compare the ICL with LASIK (or other keratorefractive techniques) in terms of refractive correction. 
Sanders and Vukich compared the results of 1,678 LASIK eyes with 144 $\mathrm{ICL}$ eyes for correction of myopia between -4 and $-7.88 \mathrm{D}$. Best spectaclecorrected visual acuity (BSCVA) loss of at least two lines was significantly higher in the LASIK eyes in the early post-operative period, whereas a BSCVA gain of at least two lines was statistically higher with the ICL at one and six months post-operatively. The proportion of cases seeing 20/15 or better, as well as 20/20 or better, at six months post-operatively was higher in the ICL group. Correction with ICL was statistically more predictable, and stability of refraction was also significantly better in the ICL group. ${ }^{13}$

By comparing 164 LASIK eyes with 164 ICL eyes matched for age, gender and mean level of pre-operative spherical-equivalent refraction, Sanders also found that ICL performed better than LASIK in almost all measures of safety, efficacy, predictability and stability. Figure 1 shows the postoperative spherical-equivalent refraction outcome results for the ICL and LASIK groups at six months post-operatively in this study; the ICL group had significantly more cases within $\pm 0.50 \mathrm{D}$ (91 versus $67 \%$; $<<0.001$ ) and $\pm 1.00 \mathrm{D}$ (99 versus $88 \% ; p<0.001$ ) of emmetropia than the LASIK group. ${ }^{14}$ Figure 2 compares the uncorrected visual acuity (UCVA) after ICL implantation and LASIK with the pre-operative BSCVA. For correction of moderate to high myopia, the same authors compared 559 LASIK eyes with 210 ICL eyes operated on for myopia between -8 and -12D. Again, every index of BSCVA, UCVA, predictability and stability of refraction favoured the ICL over LASIK. ${ }^{15}$ The three above-mentioned studies involved ICL eyes included in the 14-site FDA clinical trial for ICL myopic correction, and were followed up for six or 12 months post-operatively.

Tsiklis et al. compared one eye of a patient treated with LASIK for high myopia (spherical-equivalent refraction of $-9.75 D ; 5 \mathrm{~mm}$ optical zone) with the contralateral eye treated with an ICL (spherical-equivalent refraction of -9.50D). At nine years post-operatively, mean sphericalequivalent refraction was $-1.00 \mathrm{D}$ in the eye with the $\mathrm{ICL}$ and $-1.75 \mathrm{D}$ in the eye that underwent LASIK. Refraction correction regressed in the LASIK eye during the first six post-operative months. UCVA was 20/25 in the eye with the ICL and 20/30 in the LASIK eye. The latter experienced more night-vision problems (glare and halos). Although the patient initially preferred the LASIK procedure, increased overall satisfaction was reported for the ICL eye during the follow-up period. ${ }^{16}$

In a non-peer-reviewed publication, Turner highlighted the fact that myopic correction results are better with the ICL in comparison with LASIK, especially in high myopes, who are at a higher risk of complications such as corneal ectasia, halo, glare and overall night-vision symptomatology. ${ }^{17}$ In another non-peer-reviewed publication, Neatrour described a retrospective study involving 79 consecutive eyes undergoing vision correction for high myopia. Eighteen eyes had ICLs (average age 34 years; range 22-45 years) and 61 eyes had LASIK (average age 39 years; range 23-61 years). ICL eyes had superior results in comparison with LASIK eyes in terms of UCVA, safety, accuracy within $0.05 \mathrm{D}$ and contrast sensitivity. Wavefront analyses revealed an increase in root mean square, coma and spherical aberration over time in LASIK eyes, whereas root mean square and coma remained consistent and spherical aberration decreased in the ICL eyes. ${ }^{18}$

Another retrospective study to compare the outcomes of ICL versus LASIK to correct high myopia (range -10.0 to -23.0D) was performed by Hrubá et al. LASIK was performed in 70 eyes of 48 patients with pre-operative spherical-equivalent average of $-12.9 \pm 3.8 \mathrm{D}$ and average astigmatism of
Figure 1: Six Months Post-operative Spherical-equivalent Manifest Refraction Graphs for the Implantable Collamer Lens and Laser-assisted In Situ Keratomileusis Series

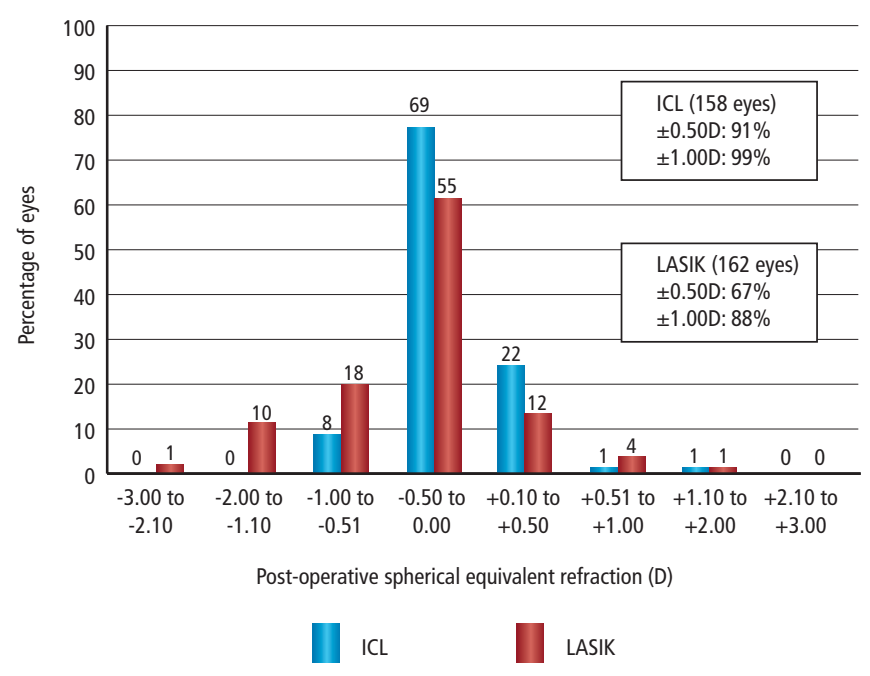

Figure 2: Comparison of Pre-operative Best Spectacle-corrected Visual Acuity with Uncorrected Visual Acuity Six Months After Implantable Collamer Lens Implantation (A) and Laser-assisted In Situ Keratomileusis (B)

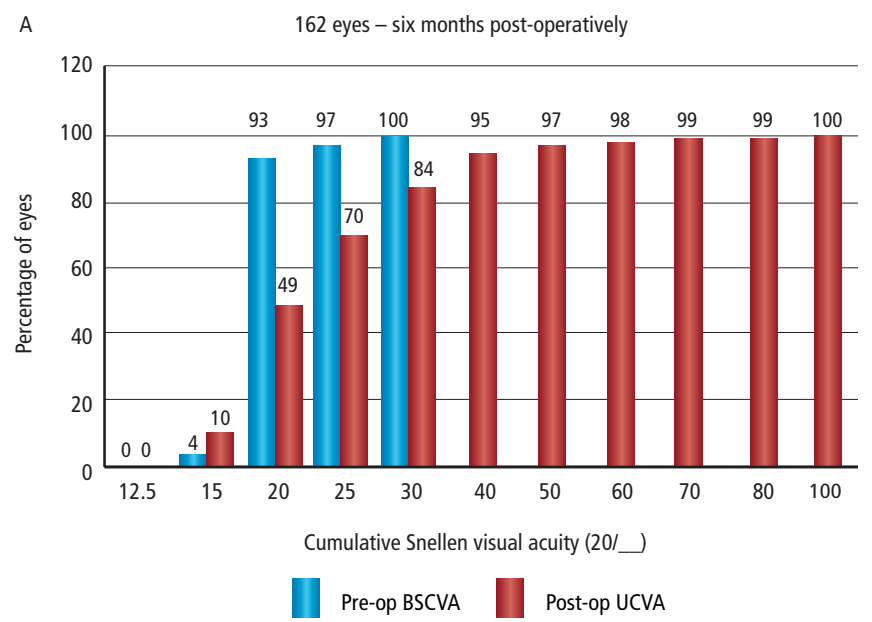

B

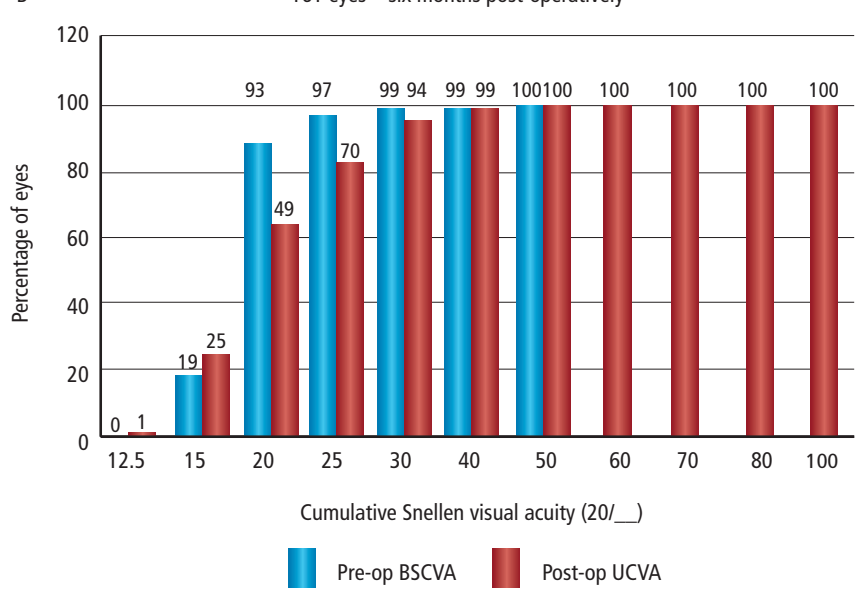

$-0.99 \pm 1.2 \mathrm{D}$. This group was divided into two subgroups: for 46 eyes, the target was emmetropia; for the other 24 eyes, residual myopia was the target. ICLs were implanted in 36 eyes of 21 patients with a pre-operative spherical-equivalent average of $-15.21 \pm 4.0 \mathrm{D}$ and average astigmatism of 
Table 1: Comparison of Laser-assisted In Situ Keratomileusis and Implantable Collamer Lens

\begin{tabular}{lll} 
& $\begin{array}{l}\text { Implantable Collamer } \\
\text { Lens (ICL) }\end{array}$ & $\begin{array}{l}\text { Laser-assisted In Situ } \\
\text { Keratomileusis (LASIK) }\end{array}$ \\
\hline Cornea & Untouched & $\begin{array}{l}\text { Modified shape and } \\
\text { biomechanics }\end{array}$ \\
\hline Tear film & Untouched & Decreased (dry eye) \\
\hline Crystalline lens & $\begin{array}{l}\text { ICL vaulting over } \\
\text { the crystalline lens }\end{array}$ & Untouched \\
\hline Visual recovery & Fast & Delayed (flap and \\
& corneal healing \\
\hline HOA & Low & Induced HOA \\
\hline CSF pressure & $\begin{array}{l}\text { Unchanged or } \\
\text { increased }\end{array}$ & Decreased \\
\hline IOL calculation for cataract & Unchanged & Less predictable outcome \\
\hline Retinal image magnification & Increased & $\begin{array}{l}\text { Less increase compared } \\
\text { with ICL }\end{array}$ \\
\hline Effective optical zone & 16-25\% gain & $\begin{array}{l}\text { Limited for same amount } \\
\text { of myopia }\end{array}$ \\
\hline Predictability & Statistically more & $\begin{array}{l}\text { Less predictable (healing } \\
\text { predictable }\end{array}$ \\
\hline Stability & Significantly better & Regression and less stability \\
\hline CSF = cerebrospinal fluid; HOA $=$ hypertrophic osteoarthropathy; IOL = intraocular lens.
\end{tabular}

$-0.92 \pm 0.69 \mathrm{D}$. This group was also divided into two subgroups: for 25 eyes, the target was emmetropia; for the other 11 eyes, residual myopia was the target. Three years after surgery in the emmetropia group, the average post-operative spherical equivalent was $-0.99 \mathrm{D}$, with reduction of myopia by $92.0 \%$ with LASIK. The corresponding values for the ICL group were $-0.52 \mathrm{D}$ and $96.1 \%$. In the group with residual myopia as the target, the average post-operative spherical equivalent was -1.74D, with reduction of myopia by $86 \%$ with LASIK. The corresponding values for the ICL group were $-1.58 \mathrm{D}$ and $90.7 \%$. Regarding final post-operative BSCVA, 1.7, 17.1, 55.7 and $22.9 \%$ of the LASIK eyes lost two Snellen lines, lost one line, were unchanged or improved by one line, respectively. The corresponding values for the $\mathrm{ICL}$ eyes were $0,0,2$ and $72.3 \%$ Re-operation was necessary in $17.1 \%$ of the LASIK eyes, and in $2.7 \%$ of the ICL eyes. ${ }^{19}$

Table 1 summarises the main differences between ICL implantation and LASIK for the correction of myopia.

\section{Hyperopic Correction}

For hyperopia correction, Horáková et al. compared 37 LASIK eyes of 20 patients with an average age of $36.3 \pm 11.8$ years and followed up for $28.1 \pm 10.2$ months with $21 \mathrm{ICL}$ eyes of 13 patients with an average age of $28.6 \pm 6.1$ years and followed up for 30.4 \pm 20.9 months. Better UCVA was obtained with the ICL, and BSCVA improved only after ICL implantation. Post-operative refraction stability was also better with the ICL, while continuous regression was evident in the LASIK group. ${ }^{20}$ The same authors also demonstrated higher efficiency and safety after ICL implantation during the entire follow-up period, and higher subjective patient satisfaction was determined, using a questionnaire, in the $\mathrm{ICL}$ group. ${ }^{2}$

\section{Astigmatic Correction}

For astigmatic correction, Schallhorn et al. performed a prospective, randomised study consisting of 43 eyes implanted with the toric $\mathrm{ICL}$ and 45 eyes receiving photorefractive keratectomy (PRK) with mitomycin C to correct myopia from -6.00 to $-20.00 \mathrm{D}$ with astigmatism from 1.00 to 4.00D. The toric lens performed better than PRK in all measures of safety, efficacy, predictability and stability in this study. Mean BSCVA, change in BSCVA, proportion of cases with improvement of one or more lines of BSCVA, proportion of cases with BSCVA and UCVA of 20/12.5 or better and of $20 / 16$ or better and predictability within $\pm 1.00 \mathrm{D}$ were all significantly better in the toric $\mathrm{IOL}$ group at all time periods studied postoperatively (one day, one week, and one, three, six and 12 months). Similarly, photopic and mesopic contrast sensitivity was significantly better at all post-operative time-points with the toric ICL.22

\section{Summary}

Over the last decade, the ICL has undergone various stages of innovation and development, surgical techniques have significantly improved and more information has become available on the long-term results of these techniques. Available clinical studies comparing the $\mathrm{ICL}$ with keratorefractive procedures for the correction of different refractive errors demonstrate the advantages of the ICL in practically all measures of safety, efficacy, predictability and stability. The excellent predictability and efficacy combined with the low incidence of post-operative complications and overwhelming patient satisfaction make the ICL and the toric ICL extremely valuable tools for refractive surgeons to address the needs of their patients.
1. Applegate RA, Howland HC, Refractive surgery, optical aberrations, and visual performance, I Refract Surg, 1997;13:295-9

2. Oliver KM, Hemenger RP, Corbett MC, et al., Corneal optical aberrations induced by photorefractive keratectomy, J Refrac Surg, 1997;13:246-54.

3. Durrie DS, Lesher MP, Cavanaugh TB, Classification of variable clinical response after photorefractive keratectomy for myopia, J Refract Surg, 1995;11:341-7.

4. Colin J, Robinet A, Clear lensectomy and implantation of a lower-power posterior chamber intraocular lens for correction of high myopia, Ophthalmology, 1997;104:73-8.

5. Goldberg MF, Clear lens extraction for axial myopia, Ophthalmology, 1987;94:571-82.

6. El-Danasoury A, Posterior chamber implantable Collamer lens ICL \& toric ICL. In: Agarwal A (ed.), Refractive Surgery, Lippincott Inc., in press

7. Zaldivar R, Davidorf JM, Oscherow S, Posterior chamber phakic intraocular lens for myopia of -8 to -19 diopters, J Refract Surg, 1998;14:294-305.

8. The Implantable Contact Lens in Treatment of Myopia (ITM) Study Group, U.S. Food and Drug Administration clinical tria of the implantable contact lens for moderate to high myopia Ophthalmology, 2003;110:255-66.
9. Sanders DR, Doney K, Poco M; ICL in Treatment of Myopia (ITM) Study Group, United States Food and Drug Administration clinical trial of The Implantable Collamer Len (ICL) for moderate to high myopia; three-year follow up, Ophthalmology, 2004;111:1683-92.

10. Pesando PM, Ghiringhello MP, Tagliavacche P, Posterior chamber collamer phakic intraocular lens for myopia and hyperopia, J Refract Surg, 1999;15:415-23.

11. Sanders DR, Schneider D, Martin R, et al., Toric implantable Collamer lens for moderate high myopic astigmatism, Ophthalmology, 2007;114:54-61.

12. Sanders DR, Sarver EJ, Standardized analyses of correction of astigmatism with the Visian toric phakic implantable Collamer lens, J Refract Surg, 2007;23:649-60.

13. Sanders D, Vukich JA, Comparison of implantable contact lens (ICL) and laser-assisted in situ leratomileusis (LASIK) for low myopia, Cornea, 2006;25:1139-46.

14. Sanders DR, Matched population comparison of the Visian implantable Collamer lens and standard LASIK for myopia of -3.00 to -7.88 diopters, J Refract Surg, 2007;23:537-53.

15. Sanders DR, Vukich JA, Comparison of implantable contact lens and laser assisted in situ keratomileusis for moderate to high myopia, Cornea, 2003;22:324-31.

16. Tsiklis NS, Kymionis GD, Karp CL, et al., Nine-year follow-up of a posterior chamber phakic IOL in one eye and LASIK in the fellow eye of the same patient, I Refract Surg, 2007;23:935-7

17. Lipner M, Visian ICL edges up on LASIK for high myopes, EyeWorld, 9 October 2007.

18. Bowers LA, ICLs correct high myopia better than all-lase LASIK, Modern Medicine, 1 February 2008.

19. Hrubá H, Vlková E, Horácková M, Svacinová J, Comparison of clinical results between LASIK method and ICL implantation in high myopia, Cesk Slov Oftalmol, 2004;60:180-91.

20. Horáková $\mathrm{M}$, Vlková $\mathrm{E}$, Loukotová $\mathrm{V}$, Hlinomazová Z, Comparison of the two methods, LASIK and ICL in mild and high hyperopia correction. Part one, Cesk Slov Oftalmol, 2007;63:143-53

21. Horáková M, Vlková E, Loukotová V, Hlinomazová Z, Comparison of the efficiency and safety of the two methods, LASIK and ICL in mild and high hyperopia correction. Part two, Cesk Slov Oftalmol, 2007:63:154-64.

22. Schallhorn S, Tanzer D, Sanders DR, Sanders ML, Randomized prospective comparison of Visian toric implantable Collamer lens and conventional photorefractive keratectomy for moderate to high myopic astigmatism, J Refract Surg, 2007;23:853-67. 


\section{Editor's Recommendations}

Comparison of Implantable Contact Lens and Laser Assisted In Situ Keratomileusis for Moderate to High Myopia

Sanders R, Vukich J, Cornea, 2003:22(4):324-31.

This study aimed to compare the results of laser-assisted in situ keratomileusis (LASIK) and implantable contact lenses (ICLs) in the correction of moderate/high myopia. Five hundred and fifty-nine LASIK eyes from the Davis Duehr Eye Center, Madison, WI, and $210 \mathrm{ICL}$ eyes from the 14-site US Food and Drug Administration (FDA) Clinical Trial for ICL for Myopia were compared. These series were concurrently operated on with 8-12D of preoperative myopia and were examined at one day, one week, one month, six months and one year post-operatively. The mean baseline myopia was slightly higher in the ICL group, (ICL: $-9.8 \pm 1.7 D$; LASIK: $-9.1 \pm 0.97 D$ ). Best spectacle-corrected visual acuity (BSCVA), uncorrected visual acuity (UCVA) and refractions were collected prospectively in both series. Every index of BSCVA, UCVA, predictability of refraction and stability of refraction studied favoured the $I C L$ over the LASIK procedure. All but one of the indices (UCVA \% 20/40 or better) were statistically significant in at least half of the time periods studied. At six-month follow-up, both the loss of two or more lines (ICL: 0\%; LASIK: $2 \% ; p=0.05$ ) and gain of two or more lines (ICL: $7 \%$; LASIK: $3 \% ; p=0.04$ ) of BSCVA were better with the ICL. Similarly, efficacy outcomes with the ICL were better with predictability (attempted versus achieved $\pm 1.0 \mathrm{D}$ ) of the ICL at $90 \% ; 76 \%$ with LASIK ( $p<0.001)$. In this highly myopic series, UCVA $20 / 20$ or better was $50 \%$ with ICL compared with $35 \%$ with LASIK ( $p<0.001)$. No serious complications occurred in either series of cases. The ICL was safer and more effective than LASIK and appears to be a viable alternative to corneal refractive excimer surgery in the treatment of moderate to high myopia.

Comparison of Implantable Collamer Lens (ICL) and Laser-assisted In Situ Keratomileusis (LASIK) for Low Myopia

Sanders R, Vukich J, Cornea, 2006;25(10):1139-46.

In this study, 1,678 LASIK eyes and $144 \mathrm{ICL}$ eyes were compared, concurrently operated on with $4-7.88 \mathrm{D}$ of pre-operative spherical equivalent of the manifest refraction, and were examined at one day, one week, one month and six months post-operatively. The mean baseline myopia was slightly higher in the ICL group. BSCVA, UCVA and refractions were collected prospectively in both series. BSCVA loss of at least two lines was significantly higher in the LASIK series in the early healing period, whereas a BSCVA gain of at least two lines was statistically better with the ICL at one and six months. The proportion of cases seeing 20/15 or better uncorrected and 20/20 or better at six months post-operatively was higher in the ICL group. The ICL was statistically more predictable (attempted versus achieved) at six months; $\pm 0.50 \mathrm{D}$ and \pm 1 .0D. Stability was achieved at the one-week to one-month interval for both groups. The stability of refraction was significantly better in the ICL group than the LASIK group through six months. No serious complications occurred in either series. The ICL was safer and more effective than LASIK and seems to be a viable alternative to corneal refractive excimer surgery in the treatment of low myopia.
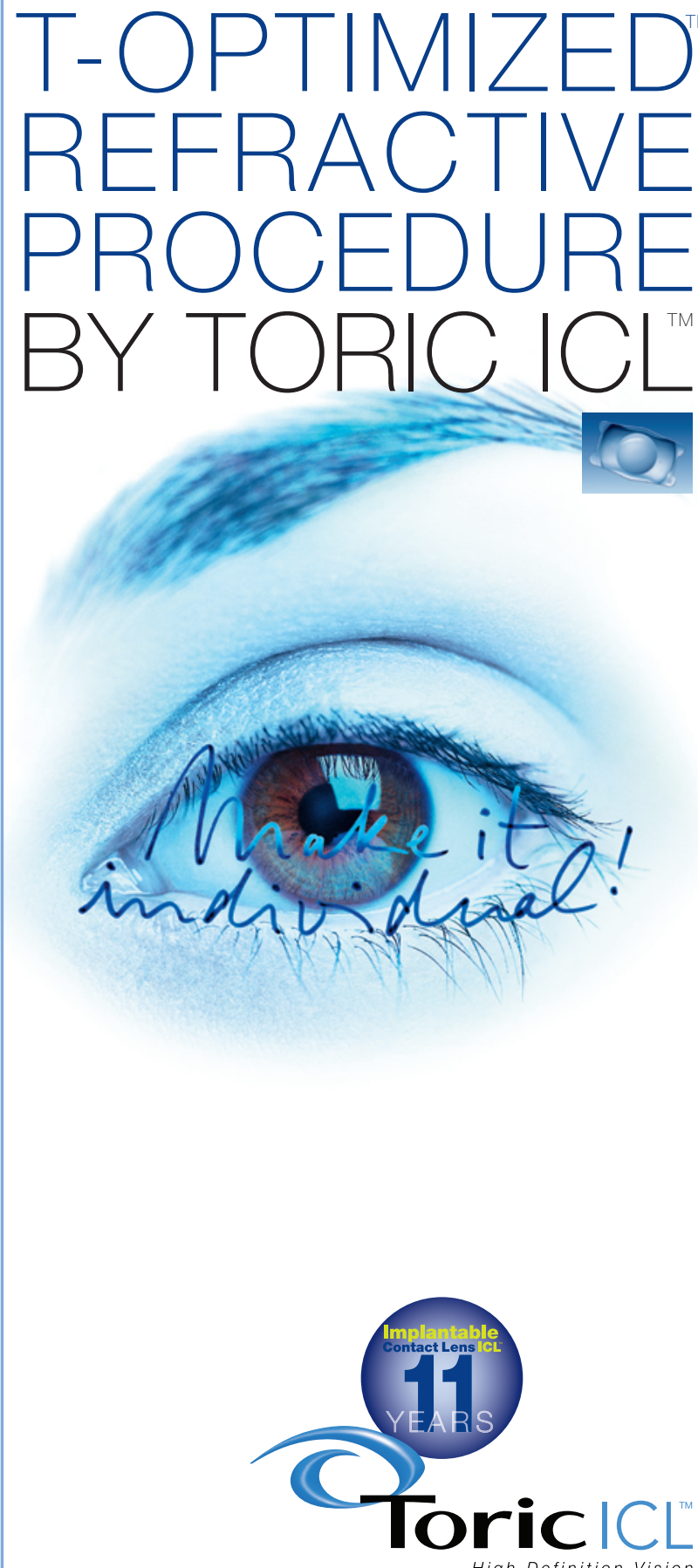

STAAR Surgical $A G$

Hauptstrasse 104, P.O. Box 463, CH-2560 Nidau, Switzerland Tel +4132 33288 88, Fax +4132 33288 99, info@staarag.ch www.staar.com

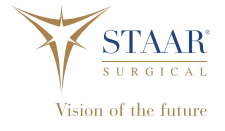

